\title{
Feature detector-level fusion methods in food recognition
}

\begin{abstract}
The analysis of dietary pattern is important in health-care to reduce the risk factors of getting diet-related chronic diseases. An automatic dietary pattern assessment via food recognition algorithm provide an alternative way to improve the overall quality of dietary pattern assessment. However, due to very high variability of food images, the fusion of multiple type of features have become inevitable. The Bag of Features $(\mathrm{BoF})$ model to encode the features are originally designed to consider a single type of features only. Therefore, this paper investigates the methods to fuse multiple features extracted from food images. Specifically, three fusion methods have been evaluated namely descriptor-level fusion, representationlevel fusion and score-level fusion. The fusion is performed on the feature detector-level between Different of Hessian (DoH) and MSER detector. Speeded-up Robust Feature (SURF) is employed for feature description. The features are encoded by using k-means clustering and Support Vector Machine with linear kernel has been employed for classification. The evaluation has been performed on UECIOO-Food dataset.
\end{abstract}

Keyword: Food recognition; Feature fusion; Bag of features 